\title{
Making Tycho Brahe's Sky Accessible to Future Astronomers
}

\section{Bertil Fabricius Dorch ${ }^{1}$, Jakob Povl Holck ${ }^{1}$, Kaare Lund Rasmussen ${ }^{1}$ and Majken Brahe Ellegaard Christensen ${ }^{2}$}

\author{
${ }^{1}$ University of Southern Denmark (SDU), \\ Campusvej 55, DK-5320, Odense, Denmark \\ email: bfd@bib.sdu.dk \\ ${ }^{2}$ Astronomicca, Odense, Denmark \\ email: info@astronomicca.com
}

\begin{abstract}
Can we make a copy of Tycho's "De Nova Stella" that can in fact survive a nova? At first, this may seem at best a nerdish, if not distinctly foolish question. However, it is also both a technological and a philosophical question: in fact, answering questions like this is linked to both technical, physical and sociological problems related to the long-term preservation and curation of objects from current and past civilizations.

The undertaking presented here is two-fold: Firstly, we report on the results from a state-ofthe art short-term project, in which we have digitized and analysed three well-known rare books pertaining to astronomical observations by Tycho Brahe on the island of Hven. The project led to the creation of free e-books, enabling open access to the sky as recorded by Tycho.

Secondly, we discuss some long-term issues related to the digital and physical preservation of scientific knowledge and heritage in general, exemplified by e.g. the works by Tycho.

Future work includes further physical analysis of the books and fragments, a systematic extraction and digitization of the astronomical observations, digital curation and dissemination, as well as research into the possibility of creating representations and replicas of the works, durable on extremely long time-scales.
\end{abstract}

Keywords. Tycho Brahe, preservation, observations, supernovae

\section{Introduction}

The preservation of scientific knowledge and cultural heritage for posterity is a multifaceted and interdisciplinary endeavour, the feasibility of which includes solving central problems of a technical, a physical and a sociological nature. Whereas the ultimate goal of the preservation of objects or works with a significance to the history and evolution of science and culture is the accessibility of content to future generations and beyond, the very long-term issues on the time-scales of civilizations or physical processes is often ignored, as the focus is on the more immediate future, assuming it to be approximated by present day society and technology.

In order to consider also the constraints put before us by physics (and astronomy), and to take a first step towards the extremely long time-scale preservation of knowledge, we ask the philosophical question, as to whether we would in principle be able to create a copy of Tycho's "De Nova Stella" that could in theory survive a nova. This is at first glance a naive question and is obviously referring to the fact that Tycho's new star was a supernova type Ia (SN 1572). However, as it is well-known, the Sun will not end as a supernova due to its relatively low mass, but rather at first as a red giant, which is likely to completely destroy most, if not all, of the innermost planets, then later fading into a 

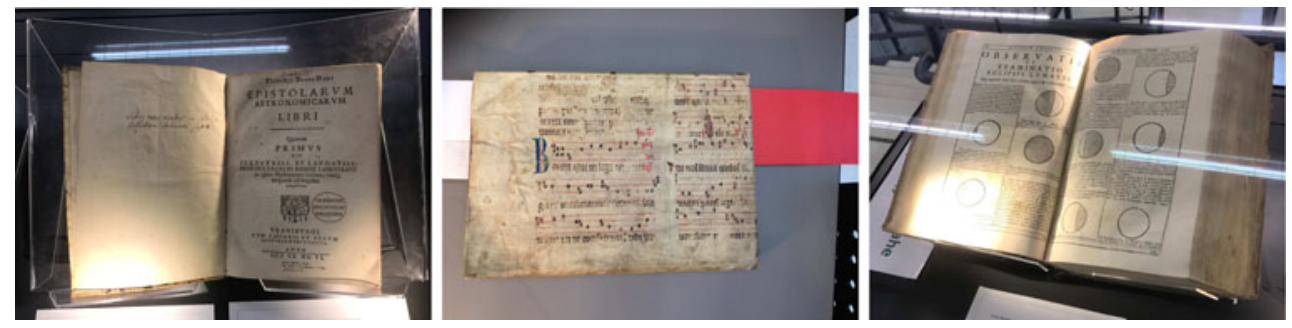

Figure 1. The three rare books, from left to right: Epistolarum Astronomicarum, De Nova Stella, and History Coelestis.

planetary nebula and most likely a white dwarf, effectively terminating life in the solar system as we know it. Ceteris paribus, the end of the Sun will also mean the end of any remnants of civilizations that will hence be lost from the inner solar system for good. The fate of the remaining parts of the solar system is more uncertain, but the violent phases of late solar evolution may also be critically dangerous to both outer solar system potential lifeforms and artifacts.

That in mind, we here report on the dissemination and curation of selected works related to the scientific discoveries of the 16th century astronomer Tycho Brahe cf. Christianson (2002). We note that Tycho Brahe is obviously famous for his discovery of the supernova in the constellation of Cassiopeia, his account of which was published in the monumental work "De Nova Stella" in 1573, one of the digitized works. Furthermore, we discuss a number of challenges and perspectives related to extremely long-term digital preservation and dissemination of cultural and scientific heritage, setting the stage for much larger philosophical questions.

\section{The Tycho Project}

We have digitized and analyzed three rare books that provide a unique insight into how astronomical observations were recorded at Tycho Brahe's observatory on the island of Hven, as well as information on the scholarly communication among astronomers in Europe at the time of Tycho Brahe.

The rare astronomical books (cf. Fig. 1) are History Coelestis (Curtz 1672), Epistolarum Astronomicarum (Brahe 1596), and a unique copy of De Nova Stella (Brahe 1573) from the rare book collection of his relative, Karen Brahe, loaned to us by Roskilde Library and Roskilde Convent, the former St Catherine's Priory.

Besides creating freely available and searchable digital e-books of the three books in ePUB format, enabling in effect easy and open access to the sky as recorded by Tycho, we have performed a non-destructive X-ray fluorescence (XRF) spectrometric analysis of one of the books, "Epistolarum Astronomicarum" containing a correspondence between Tycho Brahe and Wilhelm of Hessen: the book is a direct illustration of how astronomers perceived the sky in the late 16 th century.

This copy of "Epistolarum Astronomicarum Libri Primus" is particularly interesting because it was in fact printed on Tycho's Hven at the astronomer's own print shop. Furthermore, the book is wrapped in an even older piece of colorful parchment dating from the Middle Ages, a so-called fragment. Based on the XRF analysis we are able to draw conclusions on the composition of the fragment, which turns out to contain substantial amounts of e.g. mercury $(\mathrm{Hg})$, cf. Figs. 2 and 3.

The copy of "De Nova Stella", which is kept in the Karen Brahe Collection in Roskilde, has been digitized using mounted high resolution Canon cameras at The University Library of Southern Denmark, SDU. The digital copy has subsequently been converted into a pdf file as well as an ePUB document. 


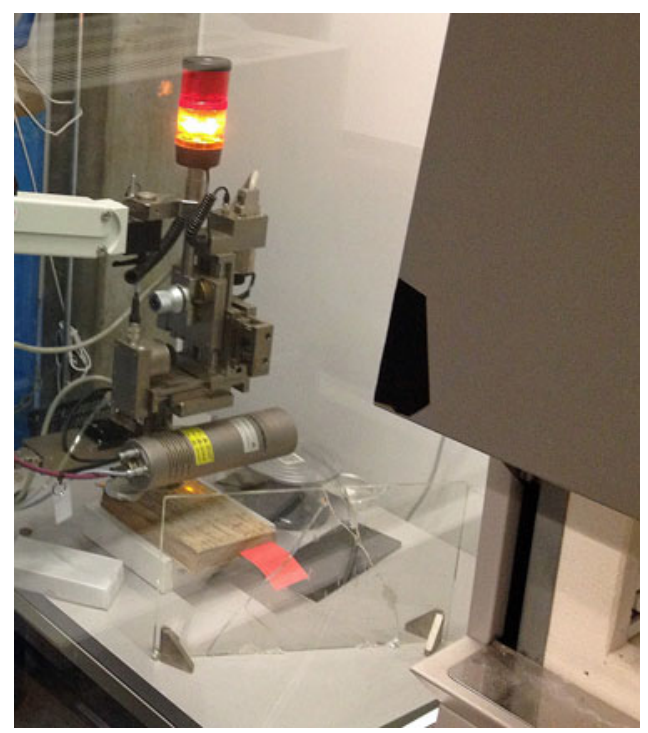

Figure 2. XRF analysis of the copy Epistolarum Astronomicarum printed on Hven: the lab setup.

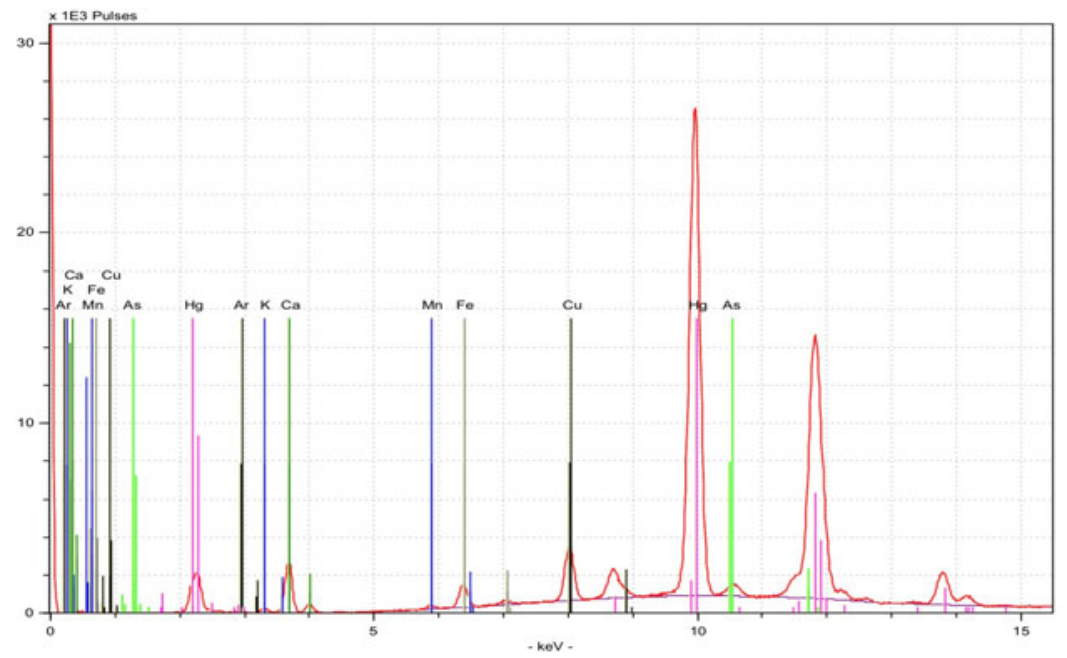

Figure 3. Spectrum of the red ink on a fragment covering the front page.

Both the "Epistolarum Astronomicarum Libri Primus" (Brahe 1596) and the posthuman edition "Historia Coelestis" (Curtz 1672) were scanned using a German-made, high-quality Zeutschel scanner also at the university library at SDU.

Using the university library's facilities, we are currently developing a Digital Library with facilitated storage and retrieval that will contain the digitized works. However, the e-books are also available on our website, at the URL www.sdu.dk/brahe.

While the short-term goals of our project was the digitization, digital dissemination, and simple paper-based reproduction of a collection of Tycho Brahe's works and observations, the ultimate aim is to begin to address the problem of the extremely long-term preservation of scientific knowledge.

The basic idea behind the challenge is to look beyond the current traditional preservation of cultural heritage by simple digitization, which we consider to be only a first step. 
The flat digitization of printed books yield only rudimentary and relatively volatile digital copies that require continued resource and priority to be maintained for prosperity. Instead, we wish to consider how the early cultural heritage and historical science can be made accessible, not only to current and future generations, but to future civilizations. In other words, what technologies are necessary to bring e.g. Tycho's observations and all subsequent reasoning by Kepler, Galileo, Newton etc. with us to our distant descendants or even to future worlds?

\section{Discussion and perspectives}

A fundamental issue faced by digital preservation is so called bit-rot caused by quantum mechanical effects that over time corrupt the original digital representation of data and converts bit values from ones to zeros and conversely. Bit-rot can occur within months cf. Cerf (2011), Hayes (1998), Kosciejew (2015) and Rosenthal (2010). The effect can be dealt with technologically by, for example, maintaining multiple copies or representations of the digitized works on multiple storage devices and periodically checking and correcting the integrity of the digital data. However, the maintenance of digitized data is relatively expensive, compared to, for example, the preservation of physical originals, cf. Balas (2010), Bote et al. (2012), Kastellec (2012), Kim (2007) and Peniston \& Langlois (2001).

An additional issue is the means to access digitally stored data: Even the authors of digital documents created early in our own era can no longer access such documents themselves due to the rapid evolution of both software and hardware standards. And even if digital data can be accessed in a distant future, the question of their intelligent interpretation still remains.

Especially, on-going changes in written and spoken languages will affect a future interpretation of the preserved information. Just how relatively fast (in astronomical terms) a language may change can be seen in the development from Old Norse to modern Scandinavian languages - as exemplified in the earliest runic texts. A modern reader without any scholarly knowledge of the runic script or the Old Norse language will be lost trying to decipher the runic inscriptions, cf. Holck (2005).

Another example is NASA's Golden Record, which was sent into space on Voyager 1 and Voyager 2, both launched in 1977. On a global scale, all humans have the advantage of a somewhat common frame of reference which is linked to the Earth's characteristic environment and firmly tied to human biology and available resources.

Generally speaking, all humans resemble each other, use similarly looking tools adapted to the human physiology etc. The observable physical world seemingly induces certain mental concepts among humans - concepts that find their way into writing and into speech, as well as art and other means communication.

Now, for an intelligent life form in the future to be able to decode an object of scientific or cultural heritage from the past, one would first of all assume that it had a sensory system similar to that of humans - i.e. the "reader" would also need mental concepts similar to those of humans concerning language and other cultural elements. Assuming that the reader has no prior knowledge of the matter, she would need some kind of semantic key to decode the recorded languages and the different symbols, in combination with the type, shape and function of the object. As in the runic example, even on Earth such a key may easily be unrecoverable.

For example, the massive Rosetta Stone, found in 1799 and kept at the British Museum, constitutes an analogue storage medium made of granodiorite, and displays the same carved text in three different writing systems. Stemming from the Hellenistic period, this decree issued in Memphis 196 BC documents the possibility of long-term information storage on physical objects. 
However, the decoding of the Egyptian writing on the Rosetta Stone was only possible due to the existence of relevant context, i.e. the recognition of culture-dependent, familiar elements on the stone, which could be correlated to the more unfamiliar elements and eventually understood.

Additionally, it seems logical to assume that digital preservation of cultural heritage data is sensitive to the political priorities of resources and to the lifetime of technological societies, while history shows that physical objects can be preserved for hundreds or even thousands of years, often independently of the rise and fall of civilizations.

\section{Conclusions}

Our long-term goal - the creation of extremely enduring copies of astronomical cultural heritage works - entails of course technological questions, but also a psychological, social and philosophical complexity. Our plan is to aim for the longest possible time-scales beyond bit-rot and beyond the timescale of civilizations - and to contemplate how we preserve our cultural heritage in the world without the now known organization and priorities of today's society; in perhaps a world without the current resources and with no memory or knowledge of current technology.

Our assumption is that digital copies will not only rot, they will become both incomprehensible, and subjected to future priorities, i.e. without power and backup, they will eventually be lost. Furthermore, the actual material and provenance of the original physical objects are seldom digitalized, if it is at all possible. In the present case of the unique copy of "De Nova Stella", classical flat digital preservation would reveal nothing about the covering fragment or any other physical constituents of the work.

We therefore conjecture that physical copies can more safely preserve our heritage, but probably not as copies of paper and ink - as something else, something new ...

Different initiatives on long term preservation have already been put into action by others: For example, the Rosetta Stone has a modern adaptation in the form of The Rosetta Disk, which can only be read by microscope. Other options include storing information in DNA for later digital retrieval, 5D glass discs etc. However, such methods require different types of equipment as well as instructions for the correct reading of the stored information. In conclusion, none of these methods are the perfect solution for delivering Tycho Brahe to future astronomers or even to survive a nova.

But rather, we can take the first small steps towards such utopia, using digitization that may eventually lead to the creation of physical storage artifacts, impervious to bit-rot and to the loss of specific software or hardware.

Our future work includes further chemical analysis of the books and fragments, as well as a systematic extraction and digitization of the astronomical observations recorded in e.g. "Historia Coelestis", and furthermore digital curation and dissemination of the works. Additionally, we intend to seek collaboration on research into the possibility of creating other types of representations and physical replicas of the works, durable on extreme time-scales, using the now available digital copies as an onset for future experiments.

\section{Acknowledgements}

We wish to acknowledge Roskilde Convent for granting us access to the copy of "De Nova Stella" from The Karen Brahe Collection. Furthermore we wish to acknowledge the SDU for providing the authors with the opportunity and facilities to conduct this project.

\section{References}

Balas, J. L. 2010, Computers in Libraries, 30 (9), 39

Bote, J., Fernandez-Feijoo, B. \& Ruiz, S. 2012, Procedia Technology, 5, 103

Brahe, T. 1573, De Nova Stella 
Brahe, T. 1596, Epistolarum Astronomicarum Libri Primus

Cerf, V. G. 2011, Proc. IEEE, 99 (6), 915

Curtz, A. 1672, Historia Coelestis

Christianson, J. R. 2002, On Tycho's Island, Cambridge University Press

Hayes, B. 1998, American Scientist, 86 (5), 410

Holck, J. P. 2005, Studies in Northern Civilization, 16, 151

Kastellec, M. 2012, Information Technology and Libraries, 31 (2), 63

Kim, T. 2007, Info. World Rev., 239, 1

Kosciejew, M. 2015, Info. Management, 49 (3), 20

Peniston, B. \& Langlois, G. 2001, Federal Computer Week, 15 (9), 50

Rosenthal, D. S. H. 2010, File Systems \& Storage, 8, (10), 1 